\title{
INJECTION OPTIONS FOR 12 GEV CEBAF UPGRADE*
}

\author{
R. Kazimi, J. F. Benesch, Y. Chao, J.M. Grames, G. A. Krafft, M. Tiefenback, B. C. Yunn, Y. Zhang, \\ Jefferson Lab, Newport News, VA 23606, USA
}

\section{Abstract}

Jefferson Lab is planning a major upgrade of CEBAF accelerator from 6 to $12 \mathrm{GeV}$. The injection energy needs to be increased accordingly from $67 \mathrm{MeV}$ to $123 \mathrm{MeV}$. While the present $100 \mathrm{keV}$ electron gun and beam formation up to $5 \mathrm{MeV}$ would remain unchanged, the accelerating SRF modules in the current injector cannot provide the desired energy increase. Two options for attaining the energy increase have been considered: (1) replacing the present injector SRF modules with new, higher gradient modules, or (2) re-circulating the electron beam through the existing cryomodules to achieve the necessary energy gain in two passes. In this paper we present computer simulation studies for these two options of the injector upgrade and list their advantages and disadvantages.

\section{INTRODUCTION}

Jefferson Lab is aiming toward a major upgrade to the Continuous Electron Beam Accelerator Facility. The maximum energy of the machine increase from $6 \mathrm{GeV}$ to $12 \mathrm{GeV}$ and a fourth experimental hall will be added. In CEBAF, the electron beam created in the injector is recirculated through two linacs up to 5 times before reaching the experimental halls. In order to keep the existing transport line, machine geometry and correct matching, the ratio of the injector energy and the energy at halls must be kept the same. However, the baseline design of the CEBAF upgrade puts the new experimental hall on the opposite corner of the CEBAF recirculation ring relative to the other experimental halls, making it a 5.5 times recirculation. Therefore, the injector energy needs to be upgraded from $67 \mathrm{MeV}$ to only $123 \mathrm{MeV}$ by less than a factor of two.

There are two options for achieving the higher injector energy. The simplest is to upgrade the accelerating modules to higher gradients without otherwise changing the present injector layout. The second option is to use the existing injector accelerating cavities twice via recirculation which maybe more cost-effective. In the following sections we will present comparative study on the stability and operability of both options using computer simulations. We start by a description of the existing CEBAF injector, description of the two injector energy upgrade options and discussion of highlights of our analysis results followed by conclusion.

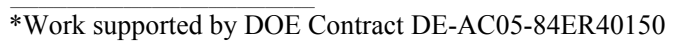

\section{CEBAF INJECTOR, BRIEF DESCRIPTION}

Figure 1 shows a schematic layout of the injector. It starts with $100 \mathrm{keV}$ photo-cathodes (only one gun is in use at any given time, the other gun being a hot spare). The next major element is the pre-buncher cavity followed by emittance limiting apertures A1 and A2 and the three-beam chopper system with three independently adjustable slits for a phase acceptance 0 to 110 ps [1]. This is the $100 \%$ acceptance aperture, not an rms or sigma value. Any beam within this window can go correctly through the bunching and acceleration process. The buncher follows and begins the main bunching of the beam. The capture section, a graded- $\beta$ five-cell cavity, provides acceleration of the beam to $500 \mathrm{keV}$. The phase and amplitude of buncher and capture cavities are crucial to the bunch compression process. A pillbox cavity after the capture section is part of a diagnostic system used to tune the bunching process through a phase compression technique [2].

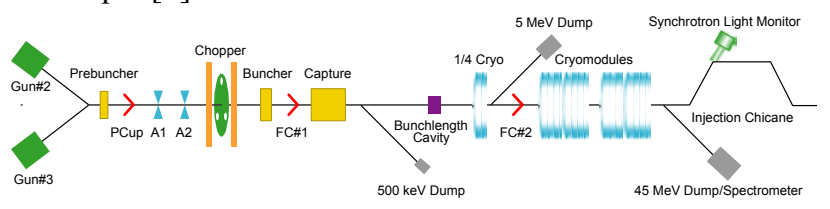

Figure1: Layout of the CEBAF Injector

Next are the first two superconducting (SRF) cavities with which the beam are further bunched and accelerated to $5 \mathrm{MeV}$. The final bunching occurs in the drift region proceeding the two accelerating modules, each containing 8 SRF cavities, which accelerate the beam to the final energy, typically 23 to $67 \mathrm{MeV}$. The beam then passes through a chicane before joining the re-circulated high energy beams in the main machine. The magnetic elements (dipoles/kicker, quadrupoles and solenoids) distributed along the injector beamline, provide beam steering, transverse focusing as well as final matching to the main accelerator. There are 3 Faraday cups in the injector to measure the beam current at different stages. Previous measurements with typical beam currents have shown a final bunch length of less than $0.3 \mathrm{~mm}$ (1 ps) and fractional energy spread of less than $10^{-3}$, both well within the requirements of the main accelerator [2].

The machine setup from gun to $5 \mathrm{MeV}$ (the quarter cryo module in figure 1) is independent of the final energy of the injector in the present machine. In both options considered for the injector energy upgrade, the section from gun to $5 \mathrm{MeV}$ remains unchanged. All changes for the upgrade are confined to the beamline after $5 \mathrm{MeV}$ and before the final injector chicane. 


\section{OPTION 1 SINGLE PASS}

The straight-through single pass option requires minimum change to the current CEBAF injector. The existing two SRF modules each consist of 8 half-meter SRF cavities with average field gradient of $7.75 \mathrm{MV} / \mathrm{m}$. To achieve $123 \mathrm{MeV}$, an average $14.75 \mathrm{MV} / \mathrm{m}$ field gradient is needed. This can be realized by refurbishing the first SRF module and replacing the second SRF module by a new high gradient one. We usually run the first few cavities of the first accelerating module at lower gradient in the present injector to lower the RF focusing effects for the not fully relativistic $5 \mathrm{MeV}$ injection beam. We would expect to do the same with the upgraded modules. We used these and other considerations in our modeling of option 1 .

\section{OPTION 2 RECIRCULATED}

In this scheme there is no need to upgrade the two accelerating modules. Instead, the $5 \mathrm{MeV}$ beam passes through the two accelerating modules twice to obtain the final energy. Figure 2 shows the recirculation design layout that replaces the beam line from $5 \mathrm{MeV}$ to the entrance of the chicane in figure 1 . The beam line includes the following sections:

1. $5 \mathrm{MeV}$ chicane into first pass cryo-modules (CM).

2. First pass CM's; top energy $64 \mathrm{MeV}$

3. Beam separator for $1^{\text {st }}$ and $2^{\text {nd }}$ pass beams

4. Recirculating arcs consisting of $445^{\circ}$ bends with extra edge focusing

5. Backleg matching sections

6. Path-length chicane (if needed) providing up to \pm 1 $\mathrm{cm}$ control of path length with roughly $\pm 3 \mathrm{~cm}$ horizontal excursion at the mid-point.

7. Reinjection into second pass CM

8. Second pass CM's; top energy $123 \mathrm{MeV}$

9. Extration from second pass CM

10. Intermediate matching for final North Linac injection (not shown)

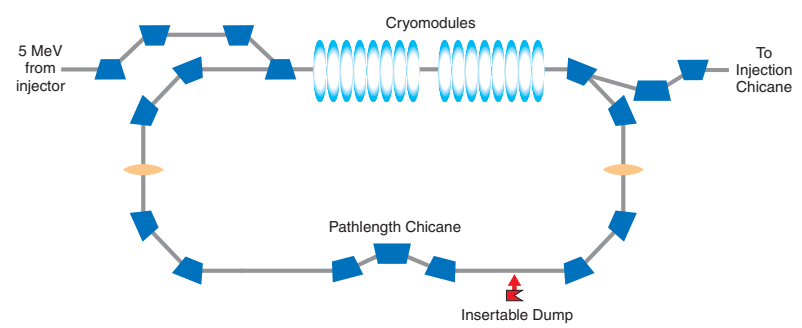

Figure 2: Schematic of the recirculation beam line.

The design and setup of the recirculated beam line maybe more complicated, however, the final beam parameters stay well within the acceptance of the main machine.

\section{COMPUTER SIMULATIONS}

The computer models of the upgraded injector for the $12 \mathrm{GeV}$ CEBAF were first built up on an OpitM deck [3] for both options. Being a simple 4D transverse matrix mapping software tool, the OptiM [4] decks allow us to design the injector beam lines and adjust their transverse optics very efficiently. Nominal parameter settings for both injector options were obtained by considering the geometric constraints of the tunnel and the transverse optics. The OptiM decks then were translated to PARMELA decks for full-scale micro particle based computer simulations. The PARMELA simulations verified the OptiM models. Moreover, PARMELA included longitudinal motion and space charge effects which were not considered in OPTIM.

Our PARMELA simulations were performed from the photocathode to the injection chicane. The cathode to $5 \mathrm{MeV}$ section was adopted from the existing PARMELA model, which was recently improved and experimentally verified [5]. The JLAB version PARMELA is based on an earlier version of the LANL PARMELA software and has been extensively customized for the CEBAF injector. The simulations were with a $100 \mu \mathrm{A} \mathrm{CW}$ electron beam with initial Gaussian distribution at cathode. 2000 micro particles were used in the simulation.

Figure 3 and 4 show the bunch length $\sigma_{z}$ and transverse beta functions $\beta_{\mathrm{x}}$ and $\beta_{\mathrm{y}}$ along the beam line for the single-pass injector scheme. The two diamonds in Figure 3 indicate the start and end of the $5 \mathrm{MeV}$ region. It shows that the bunch length achieves its final design value at the end of $5 \mathrm{MeV}$ region, before entering the two accelerating modules.

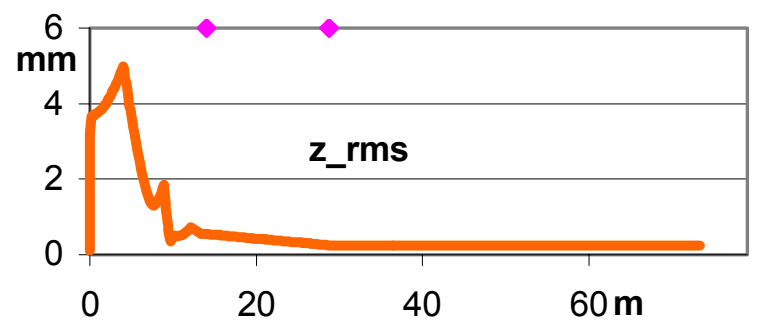

Figure 3. Bunch length from gun to Injector Chicane

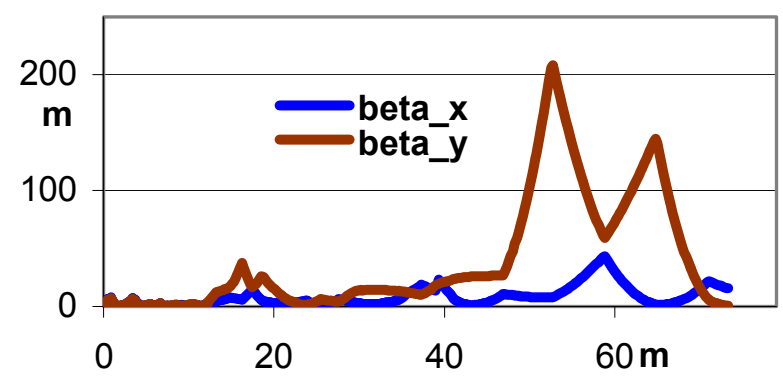

Figure 4. $\beta_{\mathrm{x}}, \beta_{\mathrm{y}}$ from gun to Injector Chicane

Figure 5 shows the bunch length $\left(\sigma_{z}\right)$ and Figure 6 is from OptiM calculations showing transverse beta function $\beta_{\mathrm{x}}, \quad \beta_{\mathrm{y}}$ and horizontal dispersion for the two-pass recirculating injector scheme. Although the recirculated beam line is relatively more complicated, the final beam parameters stay well within the acceptance of the main machine. 


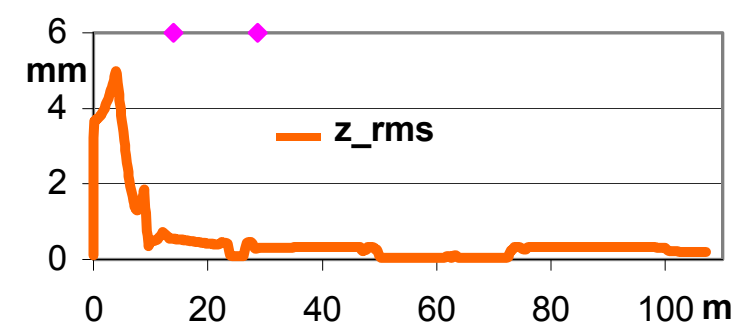

Figure 5. Bunch length from gun to Injector Chicane for recirculating option

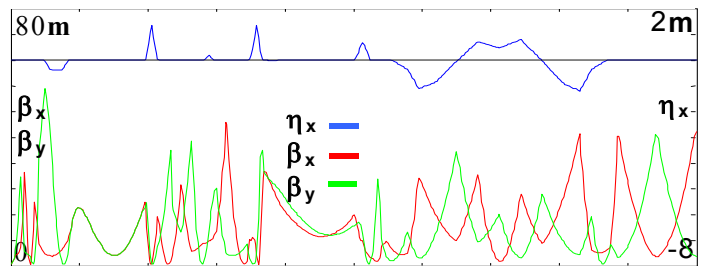

Figure 6: $\beta_{\mathrm{x}}, \beta_{\mathrm{y}}$ (left scale), $\eta_{\mathrm{x}}$ (right scale) from $5 \mathrm{MeV}$ to Injector Chicane for recirculating option

\section{SENSITIVITY STUDIES}

In order to study the two options in more detail, we performed a series of sensitivity studies by changing several injector parameters and looking at the changes to final beam parameters. We were interested in effects on the emittance, the bunch length, the energy spread, the beam ellipse parameters and the total transmission. The following cases were simulated and compared to the nominal setting for both options.

1. Energy deviation at $5 \mathrm{MeV}( \pm 30 \mathrm{keV}$ or $\pm 0.6 \%)$

2. Path length deviation (1 $\mathrm{mm}$ distributed over recirculating ring $(4 \times 0.25 \mathrm{~mm})$ )

3. Laser pulse length increased at the gun $+10 \%$

4. Laser spot size deviation (doubling the beam size at the cathode)

5. Magnetic Quad deviation (1\% random change to quads. This includes the quads in the recirculation line)

In all the cases, the total particle transmission through PARMELA remained at $100 \%$. The final beam parameters did not fall outside our limits except as follows. The recirculated design bunch length and energy spread showed significant sensitivity to energy changes at $5 \mathrm{MeV}$ and to path length changes (cases 1 and 2 above). The other parameters stayed within requirements for both single pass and recirculated options. Table 1 shows the bunch length and energy spread deviation from nominal values when the $5 \mathrm{MeV}$ energy and path length were changed.

This sensitivity of bunch length and energy spread on energy and path length changes for the recirculated option is due to the fact that the 180-degree bends in the recirculated beam line are not isochronous. The M56 matrix element generated in each of the 180-degree bends is approximately $-110 \mathrm{~cm}$. Our nominal bunch length is small, $0.2 \mathrm{~mm}$ to ensure small energy spread for user experiments. Even small changes to its value are significant. Uncompensated phase drifts or other timing errors generate additional energy spread in the bunch. A large M56 value couples with this energy spread to increase the bunch length in the 180-degree bends. Also, a large M56 tightens the requirement on SRF energy fluctuations. We are considering different ways of correcting this problem. We are researching a more isochronous design. Another possibility is to control RF phase drifts by using better diagnostics and extending an existing system used in the main CEBAF accelerator to control Rf phase movements.

Table 1: Sensitivity of bunch length and energy spread to deviations from nominal

\begin{tabular}{|l|c|c|c|c|}
\hline \multirow{2}{*}{} & \multicolumn{2}{|c|}{ Single Pass } & \multicolumn{2}{c|}{ Recirculated } \\
\cline { 2 - 5 } & $\begin{array}{c}\sigma \mathrm{z} \\
(\mathrm{mm})\end{array}$ & $\begin{array}{c}\delta \mathrm{E} / \mathrm{E} \\
\left(10^{-4}\right)\end{array}$ & $\begin{array}{c}\sigma \mathrm{z} \\
(\mathrm{mm})\end{array}$ & $\begin{array}{c}\Delta \mathrm{E} / \mathrm{E} \\
\left(10^{-4}\right)\end{array}$ \\
\hline Nominal & $\mathbf{0 . 2 4}$ & $\mathbf{1 . 3}$ & $\mathbf{0 . 2}$ & $\mathbf{2 . 9}$ \\
\hline$+30 \mathrm{keV}$ at $5 \mathrm{MeV}$ & 0.24 & 2.6 & 0.16 & 8.3 \\
\hline$-30 \mathrm{keV}$ at $5 \mathrm{MeV}$ & 0.23 & 0.5 & 0.4 & 20 \\
\hline Path $+1 \mathrm{~mm}$ & 0.24 & 0.5 & 0.41 & 1.6 \\
\hline Laser pulse $+10 \%$ & 0.22 & 1.2 & 0.19 & 3.1 \\
\hline Laser spot doubled & 0.23 & 1.3 & 0.19 & 2.9 \\
\hline Quad $1 \%$ random change & 0.24 & 1.2 & 0.19 & 2.9 \\
\hline
\end{tabular}

\section{CONCLUSION}

The CEBAF $12 \mathrm{GeV}$ upgrade requires nearly doubling the existing injector beam energy. We have examined two designs that achieve this goal. One requires upgraded accelerating modules and the other requires a more complex recirculating beam line but appears less costly. We compared the two designs using computer modeling to make sure that both designs are technically viable. The single pass design performed very much the same as the existing lower energy injector design. The recirculated design was more complicated but the final beam parameters were within specifications. Sensitivity studies showed that for recirculated design, the final bunch length and energy spread are significantly more sensitive to drifts in RF phase or amplitude. This was a consequence of the large M56 in the recirculation line. While it appears feasible to extend existing diagnostic techniques to beam based feed back control of these drifts, we are studying some changes to our design that can potentially reduce the M56 value in the recirculated injector. We are also assessing the operability of the recirculation option.

\section{REFERENCES}

[1] R. Abbott, et al, Proceedings of the 1994 International Linac Conf. Volume 2, p.777

[2] R. Kazimi, et al, Proceedings of the 2000 LINAC Conference. Volume 1, p.125-127

[3] Y-C Chao, JLAB-TN-05-003

[4] http://www-bdnew.fnal.gov/pbar/organizationalchart /lebedev/OptiM/optim.htm

[5] Y. Zhang, et. al, Proceeding of EPAC 2003 